\title{
Point Spread Function of Asymmetrically Apodized Optical Systems with Complex Pupil Filters: The One-Dimensional Case with Slit Aperture
}

\author{
M. Keshavulu Goud ${ }^{a, *}$, R. Komala ${ }^{b}$, A. Naresh Kumar Reddy ${ }^{b}$ \\ AND S. LACHA GOUD ${ }^{b}$ \\ ${ }^{a}$ Department of Physics, Nizam College, Osmania University, Hyderabad - 500 001, India \\ ${ }^{b}$ Department of Physics, College of Science, Osmania University, Hyderabad - 500 007, India
}

(Received March 27, 2011; revised version November 7, 2011; in final form January 22, 2012)

\begin{abstract}
Aimed at high resolution, an asymmetric point spread function has been obtained with good and bad sides by introducing three level asymmetric pupil functions. The good side has decreased side lobes while the bad side has increased side lobes. The asymmetry has been found to increase with the width of the edge strips within the slit aperture. On the other hand, there does not appear dependence of the asymmetry of the point spread function on the degree of amplitude apodization. But the magnitude of reduction of optical side-lobes is found to increase to a great extent by the amplitude apodization.
\end{abstract}

PACS: 42.25.Fx, 42.30.Kq, 42.79.Ci

\section{Introduction}

Apodization is the deliberate modification of the transmittance function of an optical system such that the system impulse response does not show ringing. This definition is now broadened to include any method that changes the performance of an optical system by modifying its transmittance [1]. There has been a considerable amount of studies involving apodization for various purposes viz. improvement of encircled energy [2], two-point resolution of the optical system [3], to reduce the amount of ringing present in the resulting instrumental line shape (ILS) [4], reduction of edge-ringing and edge-shifting with coherent illumination [5], reduction of the effects of aberrations [6], etc. An excellent review on the subject of apodization was presented by Jacquinoh and Roizen-Dossier [7] as early as 1964. A more or less complete account of the progress made so far in the area of apodization can be found in the work of Mills and Thompson [1].

In all the studies mentioned above, the point spread function was perfectly symmetrical in respect of positions of the minima, positions and peak intensity values of various maxima etc. In 1991 Cheng and Siu [8] employed asymmetric apodization and succeeded in obtaining the so-called "good side" with very low side-lobes and sharp central peaks and the so-called "bad side" with enhanced side-lobes and broader central peaks. It is obvious that the good side has been obtained at the cost of the bad side. In further continuation of their work [9] they obtained improved side-lobe suppression. Kowalczyk et al.

\footnotetext{
* corresponding author; e-mail: mattakgoud@osmania.ac.in, mattakgoud@hotmail.com
}

[10] used this technique to achieve axial and lateral superresolution in confocal scanning systems and Yang and Kostinski [11] applied the same technique to detect the extrasolar planets. It has been proved that, for high-reflectivity linearly chirped fiber gratings, significant improvements in the linearity of the grating dispersion can be achieved by employing asymmetric apodization technique [12].

It is well known that through proper apodization in the exit pupil of an optical system, the point spread function (PSF) at focal plane may be shaped into a desired form giving rise to superresolution over a confined region of the field of the instrument. This is the basis for our investigation. We were further motivated by the work of Cheng and Siu [8], who introduced the concept of asymmetric apodization for enhancing the resolving power of an optical system. Based on the deeper look into the mechanism of apodization, we have found that asymmetric apodization could result in better characteristics, because it has stronger suppression effect on side lobes along with narrowing central peak. In addition, the work reported on asymmetric apodization suggested to us the idea of an amplitude apodization to introduce in a central region of an aperture. This led to consideration of complex pupil functions to perform asymmetric apodization to promote the side lobe suppression and narrowing central lobe simultaneously.

For the sake of simplicity, we begin with the one-dimensional case (slit pupil) and generalize to the two dimensions (circular pupil) in the next communication. A slit aperture, which is mathematically simpler, is useful in relation to cylindrical optical elements. The study of slit images in optics has intrinsic analytical significance and important practical applications. Slit functions or so-called Gate functions are important not only in op- 
tics, but also in various other branches of science and engineering from various considerations. We get some advantages results if we employ a slit pupil function in confocal imaging systems for the imaging of line structures $[13,14]$. As an example, the gradient of the image of a straightedge is sharper than in a traditional confocal microscope.

As an effective technique asymmetric apodization across the pupil plane yields significant improvement in the focal plane energy distribution for imaging of a faint companion of a bright object. Thus, the study of asymmetric apodization has found many applications in diverse potential fields such as confocal microscopy, spectroscopy, astronomy, communication and medical imaging. For instance, the setup of an asymmetric apodization plate at the spectrometer slit to obtain the PSF good side on alternately the right and left of the strong one facilitate to detect the presence of weak line from the close proximity of bright spectral line.

In the present paper, following Cheng and Siu, we have investigated the intensity PSF of a slit aperture with asymmetric apodization introduced in the system in the form of two narrow edge strips of certain width with opposite phase transmittances of the form $\exp (\mathrm{i} \pi / 2)$ and $\exp (-\mathrm{i} \pi / 2)$ and a central region of the aperture is covered with an amplitude apodizer. The intensity PSF has been obtained for various values of the edge strips width and the transmittance of the central region of the aperture.

\section{Theory}

Within the frame of scalar diffraction theory, the amplitude impulse response of a one-dimensional optical imaging system is the Fourier transform of the pupil function and it can be written as

$$
A(u)=\int_{-1 / 2}^{1 / 2} t(x) \exp (\mathrm{i} u x) \mathrm{d} x,
$$

where $u=k \sin \theta=\frac{2 \pi}{\lambda} \sin \theta, x$ is the coordinate in the pupil plane, $u$ is the reduced dimensionless diffraction coordinate in the image plane, $\lambda$ is the wavelength of an incident radiation and $t(x)$ is the pupil function of the optical system.

Taking into account the requirement of obtaining improved side-lobe suppression and narrowing the central peak in part of the diffraction pattern, we propose, for a one-dimensional pupil of unit width, the following phase and amplitude three-level pupil function $t(x)$ :

$$
t(x)= \begin{cases}\exp (-\mathrm{i} \pi / 2)=-\mathrm{i}, & -1 / 2 \leq x<-1 / 2+b, \\ 1-4 \beta x^{2}+4 \beta x^{4}, & -1 / 2+b \leq x \leq 1 / 2-b, \\ \exp (\mathrm{i} \pi / 2)=\mathrm{i}, & 1 / 2-b<x \leq 1 / 2,\end{cases}
$$

where $\beta$ is known as the apodization parameter and determines the degree of non-uniformity of the transmission over the specified region of the pupil. The range values it takes are $0 \leq \beta \leq 1$. It is clear that $t(r)=1$ for $\beta=0$, which implies uniform transmittance over the central region of the pupil. For non-zero values of $\beta$ the central region transmittance is non-uniform. Because of exceptionally deep reduction ability and constant working angles throughout the regions of considered edge strips, we consider the odd (antisymmetric) phase functions. Thus we consider complex three-level pupils with real amplitude transmittance central slit and complex conjugated outer edge slits (Fig. 1).

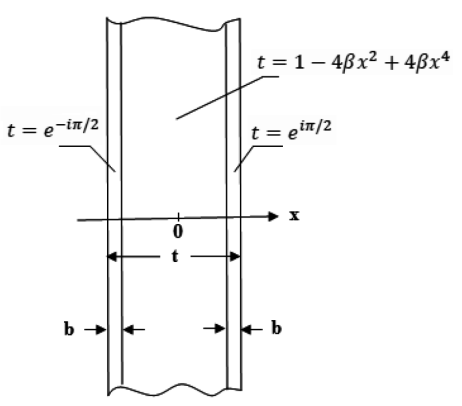

Fig. 1. General scheme of the one-dimensional asymmetric apodization function.

For chosen amplitude apodizer the amplitude transmittance is shown in Fig. 2 for different values of apodization parameter $\beta$ as a function of the pupil coordinate $x$. These show a monotonical decrease of amplitude transmittance $t(x)$ with the normalized distance $x$. At the center of the pupil $(x=0) t(x)$ is unity, i.e., highest for all values of $\beta$ and it decreases towards the edges as $x$ goes to $0.5 . t(x)$ is minimum at the edges for all values of $\beta$.

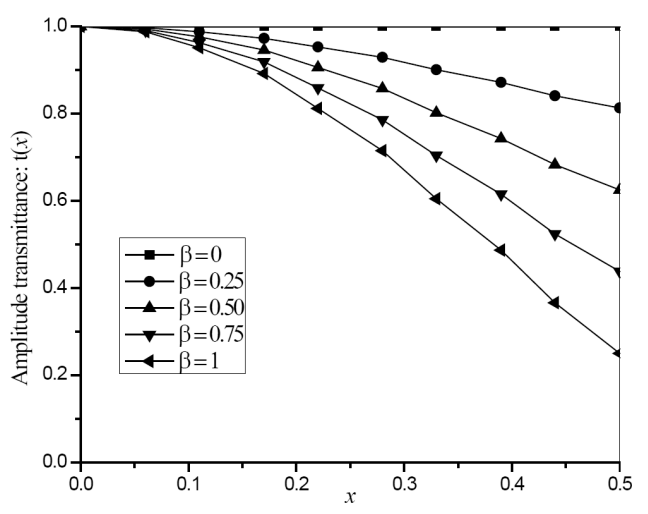

Fig. 2. Amplitude transmittance of a central region for different values of an apodization parameter $\beta$.

In this case the amplitude distribution on the focal region is equal to the sum of the three components:

(i) the diffraction field amplitude contributing by a central zone of a slit aperture of width $(1-2 b)$ :

$$
A_{0}(u)=\int_{-1 / 2+b}^{1 / 2-b}\left(1-4 \beta x^{2}+4 \beta x^{4}\right)
$$




$$
\times \exp (\mathrm{i} 2 u x) \mathrm{d} x
$$

(ii) the diffraction field amplitude contributing by the left edge strip

$$
A_{1}(u)=\int_{-1 / 2}^{-1 / 2+b}-\mathrm{i} \exp (\mathrm{i} 2 u x) \mathrm{d} x ;
$$

(iii) the diffraction field amplitude contributing by the right edge strip

$$
A_{2}(u)=\int_{1 / 2-b}^{1 / 2} \mathrm{i} \exp (\mathrm{i} 2 u x) \mathrm{d} x .
$$

Hence, the field amplitude on the image plane can be written as

$$
A(u)=A_{0}(u)+A_{1}(u)+A_{2}(u) .
$$

It means that the resultant complex amplitude distribution in the focal region is the sum of the diffraction fields $A_{1}(u)$ and $A_{2}(u)$ contributing by the narrow edge slits with opposite transmittances $-i$ and $i$ and the field $A_{0}(u)$ by the central zone covered with an amplitude apodizer.

The intensity PSF $I(u)$ which is the real measurable quantity can be obtained by taking the squared modulus of $A(u)$. Thus,

$$
I(u)=|A(u)|^{2} .
$$

\section{Results and discussion}

The results of investigations on the effects of asymmetric apodization on intensity distributions in the image plane of an optical system have been obtained from Eq. (7) as a function of diffraction coordinate $u$ varying from -12 to +12 by employing a twelve-point Gauss quadrature numerical method of integration. Gaussian quadrature [15] possesses most important desirable properties such as positivity of the weights, rapid convergence, mathematical elegance, etc. It is extremely efficient and accurate. Hence, we have chosen this method to evaluate integrals. An iterative method has been developed and applied to find the positions and intensities of first minima and maxima on either side of the diffraction center. However, we reported the results only for the good side which is the half part of the complete pattern.

We mainly focused on central-lobe shift, positions of first minima and intensity of first maxima on good side of the diffraction pattern since they are the important parameter in judging the resolution of an optical system. It may be mentioned here that we obtained these values for different cases and neglected higher order side-lobes and minima as they are suppressing fairly about to zero level. We interpreted the efficiency of asymmetric pupil functions in terms of these parameters.

The performance of the designed pupil functions is described by the asymmetric point spread functions of a slit and depicted in Figs. 3-6. In all these figures the unapodized or Airy PSF is represented by the solid curve for easy comparison. The PSFs are graphically represented in Fig. 3 for different $b$ values when the central region of the slit is unapodized. It can be seen from this figure that on the left half axis (bad side) the central peak is broadened, shifted, and lowered while on the right half axis (good side) the central peak is narrower. It tells us that the good side in which low side-lobes and a steep central peak are achieved is at the cost of worsening its counterpart. However, the magnitude of these effects depends on $b$ value. The side-lobes are reducing on both (good and bad) sides for $b=0.05$, whereas for $b=0.2$ they are increasing relative to unapodized one. The affect of amplitude filter on the suppression of secondary maxima and asymmetry in the PSF has been depicted in Fig. 4 as a function of reduced coordinate $u$ at a definite strip width $b$. As the figure reflects an asymmetry in the PSF does not depend on the degree of an apodization in a central region. On the other hand, the side-lobes suppression very much depends on it. Figure 5 is the magnified version of the region $\pi<u<4 \pi$ of Fig. 4 . It shows an evidence for amenable result of central region apodization by suppressing the side-lobes to a great extent on good side. In the presence of both antiphase and an amplitude apodization the side-lobes suppression is much larger than the only antiphase apodization. Hence, it can be emphasized that the optimum values for $b$ and $\beta$ are 0.05 and 0.5 , respectively.

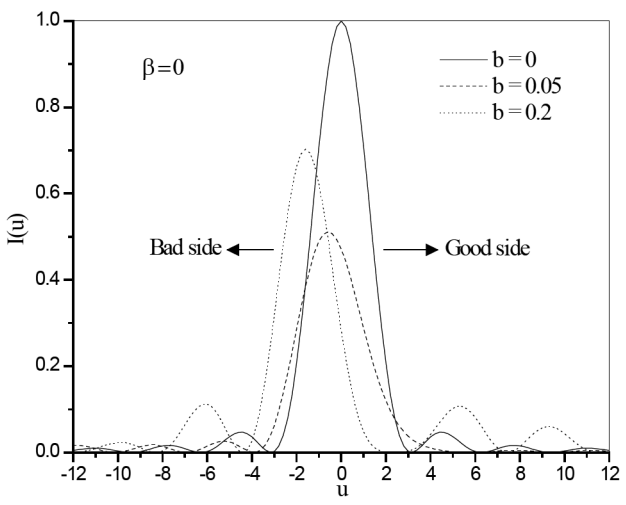

Fig. 3. Intensity profile for different $b$ values when a central region is unapodized.

In our investigation it is also observed that the central peak is narrowing and enhancing on good side when $b=0.2$ and $\beta=0.5$. In addition to this the dark region is occurring very close to the diffraction head and is extending from $u=1.6$ to 3.2 . This is accompanied by an increment of the side-lobes which is a usual effect of superresolver. These are depicted in Fig. 6.

Table I shows the intensity of first side-lobe of the diffraction pattern for different values of edge strip width $b$. These values are obtained for various amount of the apodization parameter $\beta$. It is clear from the observation that when $\beta=0$ the intensity of first side-lobe first decreases and then increases with the edge strip width $b$. As an example, when the central region is unapodized the side-lobe intensity reduces from 0.0471 to 0.0056 as 


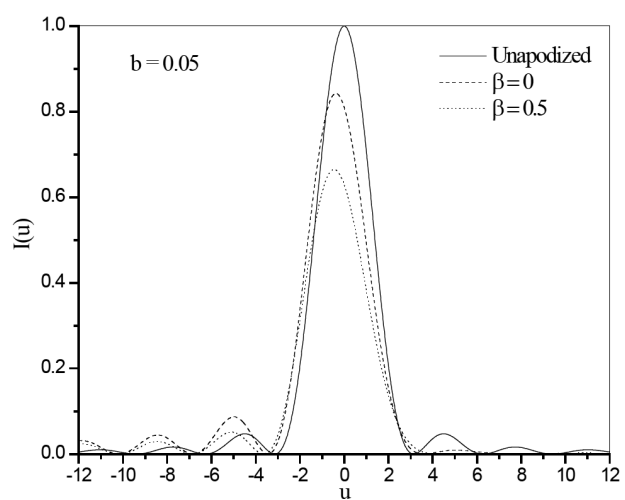

Fig. 4. Intensity profile for different amount of apodization of a central region in the presence of antiphase apodization of edge strips.

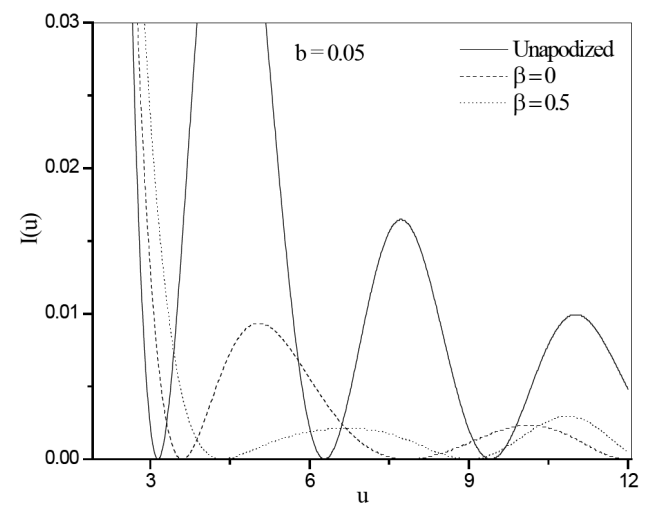

Fig. 5. Magnified version of the feet of the good side of Fig. 4.

the edge strip width increases from 0 to 0.06 and then it rises to 0.0599 as the strip width increases to 0.2 . Similar trend is noticed for all other values of $\beta$ but the maximum suppression of side-lobe is occurring at different edge strip widths for different $\beta$ values and the amount

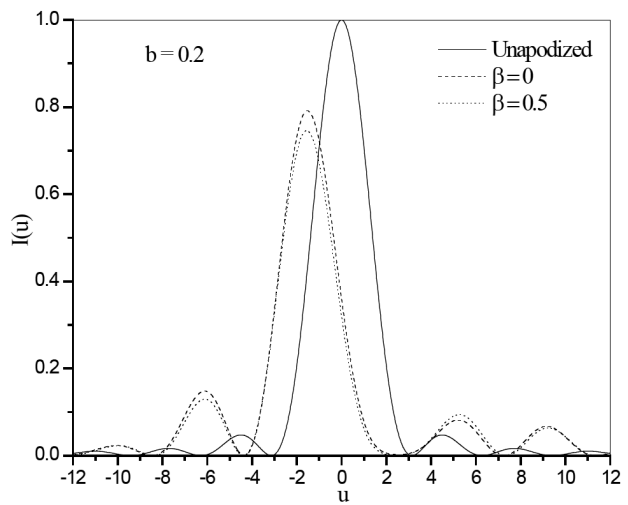

Fig. 6. Effects of edge slit width and a central region apodization on intensity profile. of reduction is increasing with the degree of apodization. However, the side-lobe reduction is at the cost of broadening of the main-lobe of asymmetric PSF. This can be seen in more detail from Figs. 7 and 8.

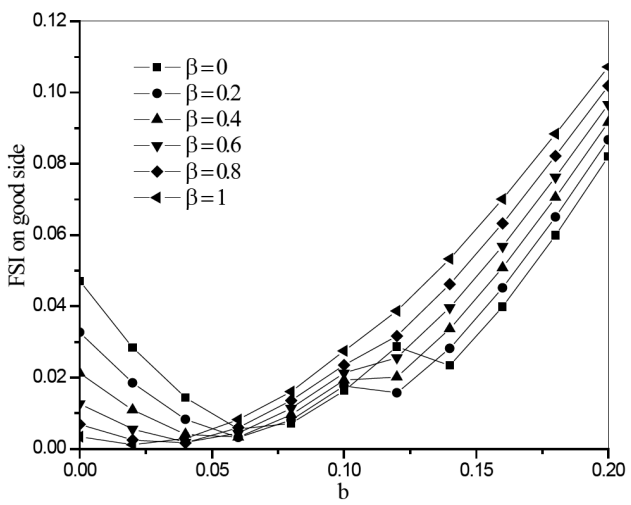

Fig. 7. Curves showing variation of first side-lobe intensity (FSI) with an edge strip width $b$ for different values of an apodization parameter $\beta$.

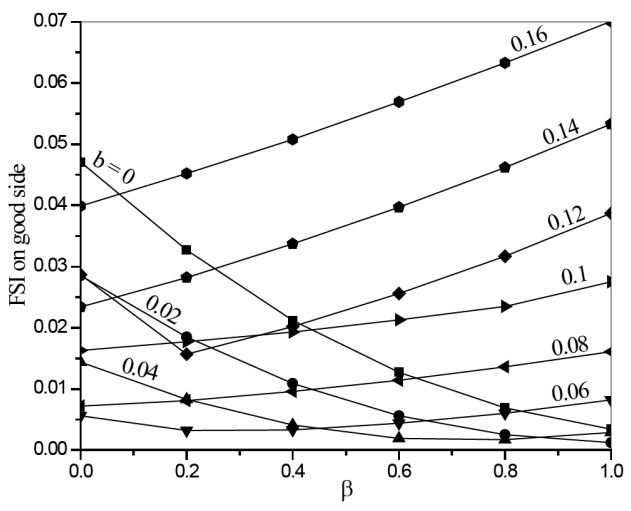

Fig. 8. Variation of FSI with an apodization parameter $\beta$ for different values of edge strip width $b$.

FSI on good side.

TABLE I

\begin{tabular}{c|c|c|c|c|c|c}
\hline \hline$b$ & $\beta=0$ & 0.2 & 0.4 & 0.6 & 0.8 & 1 \\
\hline 0 & 0.0471 & 0.0327 & 0.0212 & 0.0127 & 0.0069 & 0.0034 \\
0.02 & 0.0285 & 0.0185 & 0.0109 & 0.0056 & 0.0025 & 0.0012 \\
0.04 & 0.0144 & 0.0083 & 0.0041 & 0.0019 & 0.0017 & 0.0029 \\
0.06 & 0.0056 & 0.0032 & 0.0033 & 0.0044 & 0.0060 & 0.0082 \\
0.08 & 0.0072 & 0.0081 & 0.0096 & 0.0114 & 0.0136 & 0.0161 \\
0.1 & 0.0163 & 0.0177 & 0.0193 & 0.0213 & 0.0235 & 0.0275 \\
0.12 & 0.0287 & 0.0157 & 0.0202 & 0.0256 & 0.0317 & 0.0387 \\
0.14 & 0.0234 & 0.0282 & 0.0337 & 0.0397 & 0.0462 & 0.0533 \\
0.16 & 0.0399 & 0.0452 & 0.0508 & 0.0569 & 0.0633 & 0.0701 \\
0.18 & 0.0599 & 0.0651 & 0.0706 & 0.0763 & 0.0822 & 0.0884 \\
0.2 & 0.0820 & 0.0867 & 0.0916 & 0.0967 & 0.1019 & 0.1072
\end{tabular}

Figures 7 or 8 and listed values in Table I reveal the effect of the amplitude apodization in the central region of the aperture. As the apodization parameter $\beta$ is increasing from 0 to 0.6 , the side-lobe is suppressing from 0.0056 to 0.0033 for the same strip width $b=0.06$. For higher 
values of apodization parameter $\beta$ the maximum suppression of side-lobe is occurring at different strip widths, which are lower than the previous value. As an example, for maximum apodization $\beta=1$ the side-lobe intensity is 0.0012 and occurs at strip width $b=0.02$. This is nearly $96 \%$ lower than the value obtained when the central region is unapodized. It emphasizes that how effective is the apodization suppressing the optical side-lobes on good side. This facilitates to observe extremely faint object present very close to bright object as well as to resolve the two spectral lines which are widely varying in their intensities in the spectrum.

The position of first minimum has been evaluated for different values of the edge strip width $b$ with respect to $u=0$ and listed in Table II, for various amount of apodization parameter $\beta$. From these values it is noticed that the first minimum initially moving away from and then approaching to the diffraction center with the increase in edge strip width $b$ for all the values of apodization parameter $\beta$. Figure 9 shows this phenomenon in more detail. As $\beta$ is increasing from 0 to 1 the side-lobe is moving from 2.5165 to 2.3777 when the strip width is $b=0.2$. This implies that the spot size is reducing and subsequently narrowing the main lobe on good side of the pattern which improves the resolution in the sense of the classical criteria. This is one of the important aspects of our present study. However, the shifting of minima towards the diffraction head provides high contrast only in a narrow region on good side. This small dark zone is particularly important in the case of resolution of two line objects which are widely varying in their intensity and very close to each other. The results obtained in our investigation are clearly demonstrating that the central peak is narrowing on good side with the strip width for any given value of $\beta$. Table III shows the absolute values of first minima positions under the same conditions but with respect to central peak position. Based on this it can be said that the resolution of an optical system is degrading. The same thing may be observed from Fig. 9b. However, in the case of asymmetric apodization the center of the diffraction pattern serves as the line of demarcation between the good side and bad side.

TABLE II

FMP on good side with respect to $u=0$.

\begin{tabular}{c|c|c|c|c|c|c}
\hline \hline$b$ & $\beta=0$ & 0.2 & 0.4 & 0.6 & 0.8 & 1 \\
\hline 0 & 3.1416 & 3.2554 & 3.3977 & 3.5798 & 3.8188 & 4.1364 \\
0.02 & 3.2828 & 3.4151 & 3.587 & 3.8196 & 4.1496 & 4.623 \\
0.04 & 3.4721 & 3.6432 & 3.8834 & 4.2491 & 4.8295 & 5.4527 \\
0.06 & 3.7711 & 4.0538 & 4.531 & 5.2367 & 5.7319 & 5.9995 \\
0.08 & 4.4815 & 5.1497 & 5.6553 & 5.9561 & 6.153 & 6.2928 \\
0.1 & 5.6947 & 5.9555 & 6.1468 & 6.2945 & 6.4127 & 3.5563 \\
0.12 & 6.1955 & 3.9301 & 3.5447 & 3.3226 & 3.1411 & 2.9821 \\
0.14 & 3.2673 & 3.1383 & 3.0216 & 2.9143 & 2.8144 & 2.7206 \\
0.16 & 2.8893 & 2.8169 & 2.7481 & 2.6825 & 2.6196 & 2.5593 \\
0.18 & 2.6636 & 2.6186 & 2.575 & 2.5326 & 2.4916 & 2.4516 \\
0.2 & 2.5165 & 2.4875 & 2.4591 & 2.4313 & 2.4041 & 2.3774
\end{tabular}

Table IV lists the central maximum shifts for various values of $b$ and $\beta$. These results indicating that the central lobe is shifting away from the diffraction center

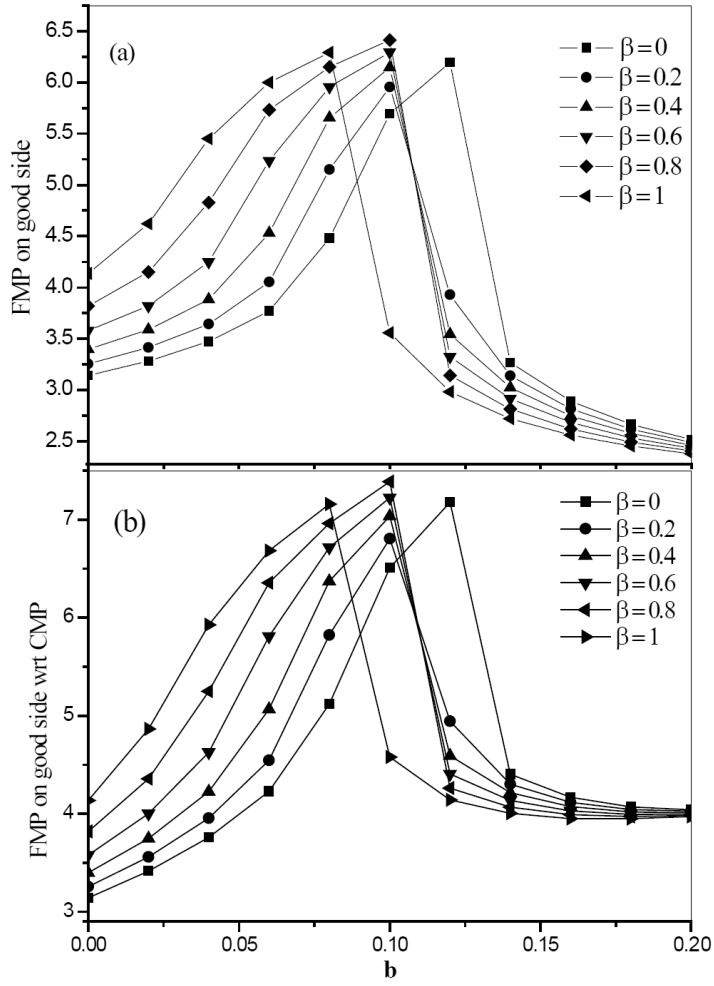

Fig. 9. (a) First minimum position (FMP) with respect to $u=0$ as a function of an edge strip width $b$ for different values of an apodization parameter $\beta$. (b) FMP with respect to central maximum position (CMP) as a function of an edge strip width $b$ for different values of an apodization parameter $\beta$.

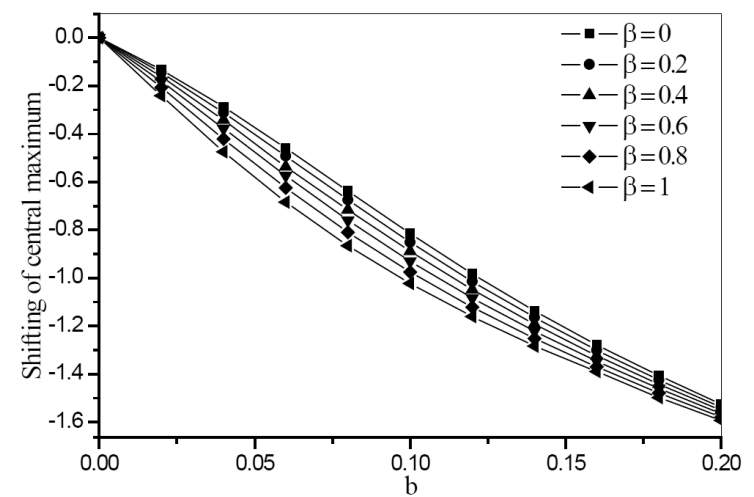

Fig. 10. Shifting of a central maximum (SCM) with an edge strip width $b$ for various amount of an apodization parameter $\beta$.

with the edge strip width $b$ whatever the amount of the apodization of the central region of the slit. This can be seen in more detail from Fig. 10. As observed from the table and figure the shift has almost a linear relation with the strip width, for all the values of $\beta$. The shift is increasing by the apodization. However, the magnitude of increase in shift depends on the degree of apodization. 
TABLE III

FMP on good side with respect to CMP.

\begin{tabular}{c|c|c|c|c|c|c}
\hline \hline$b$ & $\beta=0$ & 0.2 & 0.4 & 0.6 & 0.8 & 1 \\
\hline 0 & 3.1416 & 3.2554 & 3.3977 & 3.5798 & 3.8188 & 4.1364 \\
0.02 & 3.4148 & 3.5604 & 3.7484 & 4.0011 & 4.3568 & 4.8643 \\
0.04 & 3.7588 & 3.9551 & 4.2251 & 4.6265 & 5.2504 & 5.9274 \\
0.06 & 4.2295 & 4.5457 & 5.0682 & 5.8108 & 6.3568 & 6.6834 \\
0.08 & 5.1192 & 5.8243 & 6.3711 & 6.7164 & 6.9631 & 7.1581 \\
0.1 & 6.5094 & 6.8062 & 7.036 & 7.2251 & 7.3876 & 4.5787 \\
0.12 & 7.1779 & 4.9447 & 4.5931 & 4.4065 & 4.2623 & 4.1423 \\
0.14 & 4.4046 & 4.3028 & 4.2144 & 4.1362 & 4.0665 & 4.0039 \\
0.16 & 4.1679 & 4.1177 & 4.0716 & 4.0293 & 3.9902 & 3.9493 \\
0.18 & 4.0708 & 4.0433 & 4.0175 & 3.9932 & 3.9706 & 3.9493 \\
0.2 & 4.0409 & 4.0253 & 4.0105 & 3.9965 & 3.9832 & 3.9706
\end{tabular}

Central maximum position.

TABLE IV

\begin{tabular}{c|c|c|c|c|c|c}
\hline \hline$b$ & $\beta=0$ & 0.2 & 0.4 & 0.6 & 0.8 & 1 \\
\hline 0 & 0 & 0 & 0 & 0 & 0 & 0 \\
0.02 & -0.1320 & -0.1453 & -0.1614 & -0.1815 & -0.2072 & -0.2413 \\
0.04 & -0.2867 & -0.3119 & -0.3417 & -0.3774 & -0.4209 & -0.4747 \\
0.06 & -0.4584 & -0.4919 & -0.5372 & -0.5741 & -0.6249 & -0.6839 \\
0.08 & -0.6377 & -0.6746 & -0.7158 & -0.7603 & -0.8101 & -0.8653 \\
0.1 & -0.8147 & -0.8507 & -0.8892 & -0.9306 & -0.9749 & -1.0224 \\
0.12 & -0.9824 & -1.0146 & -1.0484 & -1.0839 & -1.1212 & -1.1602 \\
0.14 & -1.1373 & -1.1645 & -1.1928 & -1.2219 & -1.2521 & -1.2833 \\
0.16 & -1.2786 & -1.3008 & -1.3235 & -1.3468 & -1.3706 & -1.3900 \\
0.18 & -1.4072 & -1.4247 & -1.4425 & -1.4606 & -1.4790 & -1.4977 \\
0.2 & -1.5244 & -1.5378 & -1.5514 & -1.5652 & -1.5791 & -1.5932
\end{tabular}

\section{Conclusions}

We may conclude that our work, aimed at enhancing the resolution, has been carried out by considering three-level phase and amplitude pupil functions. In order to perform an asymmetric apodization the slit aperture has been divided into two narrow edge strips with opposite phase functions and the central region with non-uniform amplitude function. An asymmetric PSF is obtained with good side having reduced side-lobes and a steep central maximum. This is achieved at the cost of worsening its counterpart. The central lobe shifts to which has higher side-lobes i.e. to the bad side. The asymmetry increases with the width of the edge-strips while the asymmetry is invariable with the degree of apodization in the central region. Simultaneously suppressing the side-lobes and narrowing the main-lobe of the PSF leads to high resolution of optical systems.

In conclusion, high resolution has been achieved by performing asymmetric apodization with complex pupil filters. Their superior capabilities of side lobes suppression and narrowing of central lobe have been testified.
Unfortunately, for $b=0$ and $\beta=0.5$ values the position of first minimum is higher than the Airy case. But these values yield a better result by obtaining steep principal maximum and highly suppressed side lobes for the whole range of interest.

On the whole it can be emphasized that an additional improvement for resolution could be obtained by the use of complex pupil filters instead of phase only filters to perform asymmetric apodization. This would make the system fabrication little bit complicated but it could be a solution for cases in which a very high contrast or resolution is required. Further investigations are being carried out on this subject by considering some new parameter in addition to well defined parameters.

\section{References}

[1] J.P. Mills, B.J. Thompson, Selected Papers on Apodization: Coherent Optical Systems, SPIE Milestones series Vol. MS 119, SPIE Optical Engineering Press, Ellingham 1996.

[2] R. Barakat, J. Opt. Soc. Am. 52, 264 (1964).

[3] B.L. Mehta, J. Phys. D, Appl. Phys. 7, 329 (1974).

[4] R.J. Bell, Introductory Fourier Transform Spectroscopy, Academic Press, New York 1972.

[5] J.P. Mills, B.J. Thompson, J. Opt. Soc. Am. A 3, 704 (1986).

[6] M. Samir, R.H. Andrew, Opt. Lett. 28, 771 (2003).

[7] P. Jacquinot, B. Roizen-Dossier, Prog. Opt. 3, 29 (1964).

[8] L. Cheng, C.G. Siu, Meas. Sci. Technol. 2, 198 (1991).

[9] G.G. Siu, L. Cheng, D.S. Chiu, K.S. Chan, J. Phys. D, Appl. Phys. 27, 459 (1994).

[10] M. Kowalczyk, C.J. Zapata-Rodriguez, M. Martinez-Corral, Appl. Opt. 37, 8206 (1998).

[11] W. Yang, A.B. Kostinski, Astroph. J. 605, 892 (2004).

[12] M.N. Zervas, D. Taverner, Fiber Integrat. Opt. 19, 355 (2000).

[13] T. Wilson, S.J. Hewlett, C.J.R. Sheppard, Appl. Opt. 29, 4705 (1990).

[14] C.J.R. Sheppard, X.Q. Mao, J. Mod. Opt. 35, 1169 (1988).

[15] J.H. Mathews, K.D. Fink, Numerical Methods Using Matlab, Pearson Prentice Hall, New Jersey 2004. 